\title{
Neutrophil activation by the tissue factor/ Factor VIla/PAR2 axis mediates fetal death in a mouse model of antiphospholipid syndrome
}

\author{
Patricia Redecha, ${ }^{1}$ Claus-Werner Franzke, ${ }^{1}$ Wolfram Ruf, ${ }^{2}$ Nigel Mackman, ${ }^{3}$ and Guillermina Girardi ${ }^{1}$
}

${ }^{1}$ Hospital for Special Surgery, Weill Medical College of Cornell University, New York, New York, USA.

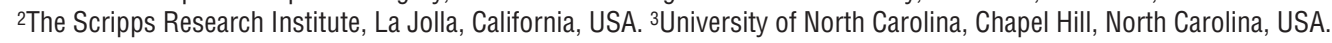

\begin{abstract}
Women with antiphospholipid syndrome (APS), a condition characterized by the presence of antiphospholipid antibodies (aPL), often suffer pregnancy-related complications, including miscarriage. We have previously shown that C5a induction of tissue factor (TF) expression in neutrophils contributes to respiratory burst, trophoblast injury, and pregnancy loss in mice treated with aPL. Here we analyzed how TF contributes to neutrophil activation and trophoblast injury in this model. Neutrophils from aPL-treated mice expressed protease-activated receptor 2 (PAR2), and stimulation of this receptor led to neutrophil activation, trophoblast injury, and fetal death. An antibody specific for human TF that has little impact on coagulation, but potently inhibits TF/Factor VIIa (FVIIa) signaling through PAR2, inhibited aPL-induced neutrophil activation in mice that expressed human TF. Genetic deletion of the TF cytoplasmic domain, which allows interaction between TF and PAR2, reduced aPL-induced neutrophil activation in aPL-treated mice. Par2 $2^{-/}$mice treated with aPL exhibited reduced neutrophil activation and normal pregnancies, which indicates that PAR2 plays an important role in the pathogenesis of aPL-induced fetal injury. We also demonstrated that simvastatin and pravastatin decreased TF and PAR2 expression on neutrophils and prevented pregnancy loss. Our results suggest that TF/FVIIa/PAR2 signaling mediates neutrophil activation and fetal death in APS and that statins may be a good treatment for women with aPL-induced pregnancy complications.
\end{abstract}

\section{Introduction}

We previously showed that inflammation is responsible for fetal injury in a mouse model of antiphospholipid antibody-induced (aPL-induced) pregnancy loss (1). We demonstrated that activation of complement, specifically generation of the anaphylotoxin $\mathrm{C} 5 \mathrm{a}$, is crucial in fetal injury induced by aPL. Tissue factor (TF) expressed on neutrophils in response to C5a contributed to the respiratory burst, trophoblast injury, and pregnancy loss induced by aPL. TF is the major cellular initiator of the coagulation protease cascade but also plays important roles in inflammation (2). Complexes of TF/Factor VIIa (TF/FVIIa) and TF/FVIIa/Factor Xa (TF/FVIIa/FXa) as well as FXa and thrombin induce proinflammatory signals by activating protease activated receptors (PARs) and inducing the expression of TNF- $\alpha$, interleukins, and adhesion molecules (3-5). In a model of microvascular inflammation, activation of PARs leads to cytokine generation and rapid induction of P-selectin-mediated leukocyte rolling $(6,7)$. In the absence of PARs, the onset of inflammation is delayed in endotoxemia and ischemia/reperfusion $(8,9)$. For many years, the antiphospholipid syndrome (APS) was considered a thrombophilic disorder, but recent human studies and mouse studies from our laboratory have shown the importance of inflammation in the pathogenesis of aPL-induced pregnancy loss (1, 10-12). Knowing the crucial role of TF and inflammation in aPL-induced pregnancy

Nonstandard abbreviations used: aPL, antiphospholipid antibody; aPL-IgG, human IgG-containing aPL; APS, antiphospholipid syndrome; DAF, decay-accelerating factor; DHE, dihydroethidium; DHR, dihydrorhodamine 123; FACS, fluorescence-activated cell sorting; FPX, fondaparinux; FRF, fetal resorption frequency; FVIIa, Factor VIIa; FXa, Factor Xa; HCV, human chromosomal vector; NH-IgG, normal human IgG; PAR, protease-activated receptor; TF, tissue factor.

Conflict of interest: The authors have declared that no conflict of interest exists. Citation for this article: J. Clin. Invest. 118:3453-3461 (2008). doi:10.1172/JCI36089. loss led us to investigate the mechanism by which TF contributes to inflammation in aPL-induced pregnancy loss. Here we demonstrated that PAR2 was upregulated in neutrophils from mice treated with human IgG-containing aPL (aPL-IgG) and that stimulation of this receptor led to neutrophil activation, trophoblast injury, and fetal death in this model of aPL-induced fetal death. We also demonstrated that statins decreased TF and PAR2 expression on neutrophils, preventing pregnancy complications in APS.

\section{Results}

Increased synthesis of TF and PAR2 in neutrophils from aPL-IgG-treated mice. We previously demonstrated increased expression of TF on neutrophils from aPL-treated mice (1). Fluorescence-activated cell sorting (FACS) analysis also revealed increased PAR2 expression on neutrophils from aPL-IgG-treated mice (aPL-IgG, 16.2\% $\pm 6.1 \%$ PAR2 positive; untreated, $6 \% \pm 2 \%$ PAR 2 positive; $P<0.05$ ). A 5.3-fold increase in PAR2 mRNA expression was observed in neutrophils from aPL-IgG-treated mice compared with untreated or normal human IgG-treated (NH-IgG-treated) mice (Table 1). In addition, immunocytochemical staining of cytospin films revealed increased PAR2 expression on neutrophils from aPL-IgG-treated mice compared with NH-IgG treated mice (Figure 1A).

To determine whether neutrophils are capable of synthesizing TF, we measured TF mRNA expression by RT-PCR in freshly isolated neutrophils from aPL-IgG-treated mice. We observed a 29.9-fold increase in TF mRNA expression in neutrophils from aPL-IgG-treated mice compared with NH-IgG-treated mice (Table 1). TF protein expression in neutrophils was also found by immunocytochemical staining with specific anti-TF mAb (Figure 1A).

PAR2 signaling is required for oxidative burst and phagocytosis in neutrophils from aPL-IgG-treated mice. Prompted by our findings of 
Table 1

$2^{-\Delta \Delta C t}$ data analysis

\begin{tabular}{|c|c|c|c|c|c|}
\hline Treatment & $\begin{array}{r}\text { Me } \\
\text { Target }\end{array}$ & $\begin{array}{l}\text { Ct } \\
\text { GAPDH }\end{array}$ & $\Delta \mathrm{Ct}$ & $\Delta \Delta \mathrm{Ct}$ & $2^{-\Delta \Delta C t}$ \\
\hline TF & & & & & \\
\hline $\begin{array}{l}\text { Untreated } \\
\text { aPL-IgG }\end{array}$ & $\begin{array}{l}33.3 \\
27.8\end{array}$ & $\begin{array}{l}22.1 \\
21.5\end{array}$ & $\begin{array}{r}11.2 \pm 1.2 \\
6.3 \pm 2.2\end{array}$ & $\begin{aligned} 0 & \pm 0.78 \\
-4.9 & \pm 0.9\end{aligned}$ & $29.9 \pm 0.7^{A}$ \\
\hline PAR2 & & & & & \\
\hline $\begin{array}{l}\text { ntreated } \\
\text { PL-IgG }\end{array}$ & $\begin{array}{l}28.7 \\
25.7\end{array}$ & $\begin{array}{l}22.1 \\
21.5\end{array}$ & $\begin{array}{l}6.6 \pm 1.8 \\
4.2 \pm 1.1\end{array}$ & $\begin{array}{r}0 \pm 1.0 \\
-2.4 \pm 1.2\end{array}$ & $5.3 \pm 0.4^{B}$ \\
\hline
\end{tabular}

Relative quantification of TF and PAR2 between polymorphonuclear neutrophils from aPL-IgG-treated and untreated mice. The comparison was performed in pairs using the same target gene and the same neutrophil samples ( $n=5$ mice per group). $\Delta \mathrm{Ct}$, mean target $\mathrm{Ct}$ minus mean GAPDH Ct; $\Delta \Delta \mathrm{Ct}$, mean $\Delta \mathrm{Ct}$ aPL-IgG-treated minus mean $\Delta \mathrm{Ct}$ untreated for the same target gene; $2^{-\Delta \Delta C t}$, normalized target gene amount relative to target gene amount in untreated mice. $\Delta \Delta \mathrm{Ct}$ equals 0 when the neutrophils of interest are used as calibrator. ${ }^{A}$ Expression increased 29.9-fold compared with untreated neutrophils $(P<0.01)$. BExpression increased 5.3-fold compared with untreated neutrophils $(P<0.01)$.

increased PAR2 expression, increased ROS production, and phagocytosis in neutrophils from aPL-IgG-treated mice, we determined whether PAR2 signaling is involved in neutrophil activation. To study the role of PAR2, we examined neutrophils from $\mathrm{Par}^{-/-}$mice. The genetic deletion of Par 2 dramatically reduced neutrophil activation in aPL-IgG-treated mice (Figure 1, B-D). ROS production was significantly reduced in neutrophils from aPL-IgG-treated $\mathrm{Par}^{-/-}$ mice compared with aPL-IgG-treated wild-type mice $(P<0.01$; Figure 1B). The aPL-IgG-induced increase in phagocytosis was also reduced in $\mathrm{Par2}^{-/-}$mice (Figure $1 \mathrm{C}$ ). In addition, neutrophils from $\mathrm{Par}^{-/-}$mice did not show an impaired capacity to generate oxidants or impaired phagocytosis when stimulated in vitro with PMA (Figure 1, C and D).

TF-dependent activity of neutrophils in aPL-IgG-treated mice is not affected by PAR1 deficiency. PAR1 deficiency did not prevent the aPL-IgG-induced increase in ROS production and phagocytosis (Figure 1, E and F). ROS production and phagocytosis in neutrophils from aPL-IgG-treated $\mathrm{Parl}^{-/-}$mice were not different from those of aPL-IgG-treated wild-type mice, which indicates that PAR1 activation is not required for neutrophil activation in this model. The finding that PAR1 was not required for aPL-induced neutrophil activation was further substantiated by our observation that hirudin - which inhibits thrombin, the main ligand for PAR1 - did not prevent neutrophil activation in aPL-IgG-treated mice (Figure 1, E and F). These results extend our previous work showing that hirudin does not prevent aPL-induced pregnancy loss (13). Neutrophil-enhanced phagocytosis and ROS production in response to aPL-IgG were also unaffected by the use of the anticoagulant fondaparinux (FPX; Figure 1, E and F). We previously reported that FPX did not prevent aPL-induced pregnancy loss (13). This suggests that generation of FXa is not required for the aPL-induced, TF-dependent increase in neutrophil activity. The fact that neutrophil activation requires neither FXa generation, thrombin generation, or PAR1 activation suggests that neutrophil activation is mediated by the TF/FVIIa/PAR2 axis.

$T F / F V I I a$ signaling is required for oxidative burst and phagocytosis in neutrophils from aPL-IgG-treated mice. To confirm the role of TF/
FVIIa/PAR2 signaling in aPL-IgG-induced neutrophil activation, we treated mice expressing human TF (hTF) with a specific $\mathrm{mAb}, 10 \mathrm{H} 10$, which selectively blocks TF/FVIIa signaling through PAR2. We also treated mice with antibody 5G9, which only prevents TF procoagulant activity, as well as a mouse IgG1 antibody as a control. Both 5G9 and 10H10 have been used previously in tumor growth xenograft models, and the prior study demonstrated that TF signaling rather than coagulation is important for tumor growth (14). Because these are human antibodies, we used them in human chromosomal vector (HCV) mice humanized for TF. HCV

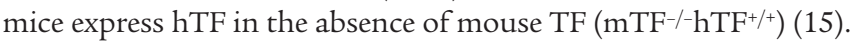
$\mathrm{HCV}$ mice showed increased TF expression on neutrophils after aPL-IgG treatment comparable to that observed in wild-type mice that express $\mathrm{mTF}$ (aPL-IgG HCV, $24.2 \% \pm 3.2 \%$ TF positive; aPL-IgG wild type, $20.3 \% \pm 2.3 \%$ TF positive). Neither increased oxidative burst nor increased phagocytosis was observed in neutrophils from $\mathrm{HCV}$ mice treated with aPL-IgG and $10 \mathrm{H} 10$ (Figure 2A). Inhibition of direct TF/FVIIa signaling through PAR2 using $10 \mathrm{H} 10$ prevented neutrophil activation, demonstrating a role for TF/FVIIa/PAR2 signaling in aPL-IgG-induced neutrophil activation. On the other hand, treatment with 5 G9 had no effect on neutrophil activation (Figure 2A). These results are consistent with our previous observation that anticoagulation does not rescue pregnancy in APS (13).

To substantiate the finding that PAR2 mediates aPL-IgG-induced neutrophil activation and to confirm that TF/FVIIa signaling is required for neutrophil activation in aPL-IgG-treated mice, we studied $\mathrm{TF}^{\Delta \mathrm{CT} / \Delta \mathrm{CT}}$ mice. These mice lack the 18 carboxyterminal amino acids of the cytoplasmic domain of TF (16). This cytoplasmic domain is involved in signaling and is required for TF/FVIIainduced cellular responses (16). TF ${ }^{\Delta \mathrm{CT} / \Delta \mathrm{CT}}$ mice treated with LPS exhibited less inflammation and are protected from lethality independent of changes in coagulation (16). We observed increased TF expression, but no increase in either oxidative stress or phagocytosis, in neutrophils from aPL-IgG-treated TF $\Delta \mathrm{CT} / \Delta \mathrm{CT}$ mice (Figure $2 \mathrm{~B})$, reinforcing the idea that TF/FVIIa signaling is required for neutrophil activation and fetal injury in aPL-IgG-treated mice. Taken together, these results suggest that interaction of TF with PAR2 is required for aPL-IgG-induced neutrophil activation.

Genetic deletion of Par2 prevents fetal loss induced by aPL-IgG. Given that the previous experiments showed the importance of PAR2 signaling in TF-mediated neutrophil activation, we investigated the role of PAR2 in pregnancy outcomes in aPL-IgG-treated mice. Consistent with previously published reports $(1,11-13)$, approximately $40 \%$ of the embryos in aPL-IgG-treated wild-type mice died $(40.5 \% \pm 5.8 \%$; Figure $3 \mathrm{~A})$, a fetal resorption frequency (FRF) more than 4-fold greater than that in NH-IgG-treated mice $(10.1 \% \pm 3.2 \%)$. This aPL-IgG-induced increase in FRF was not observed in Par2 $2^{--}$ mice. FRF in aPL-IgG-treated $\mathrm{Par}^{-{ }^{--}}$mice was not different from that of NH-IgG-treated wild-type mice (Figure 3A), which indicates that signaling through PAR2 is required for aPL-induced pregnancy loss. Moreover, genetic deletion of PAR2 reduced inflammation in deciduae from aPL-IgG-treated mice. Less complement C3 deposition was observed in deciduae from aPL-IgG-treated Par2 $2^{-1-}$ mice, comparable to deposition in those from NH-IgG-treated mice (Figure 3B). Par2 ${ }^{-1-}$ mice treated with aPL-IgG also showed less free radical-mediated lipid peroxidation in decidual tissue, comparable to that of wild-type mice treated with NH-IgG (Figure 3C). Oxidative damage in deciduae from aPL-IgG-treated $\mathrm{Par}^{-/-}$mice was minimal and comparable to that in deciduae from NH-IgG-treated wild-type mice (Figure 3C). 
A
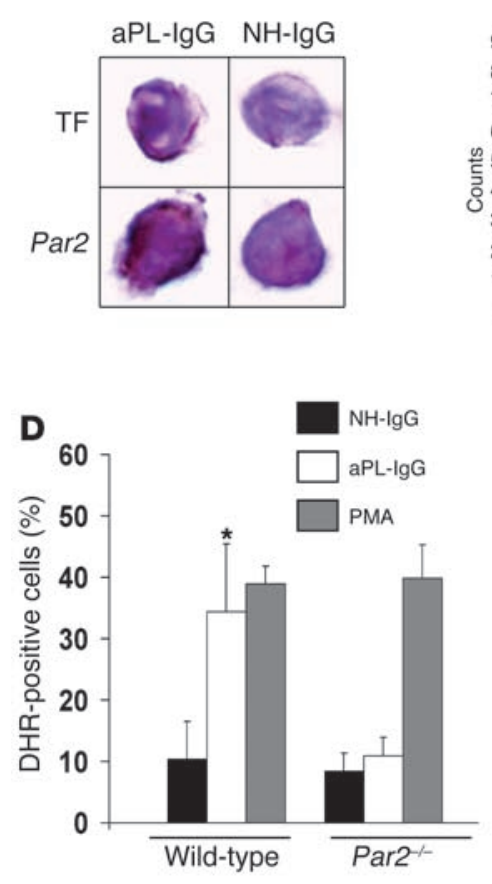

B
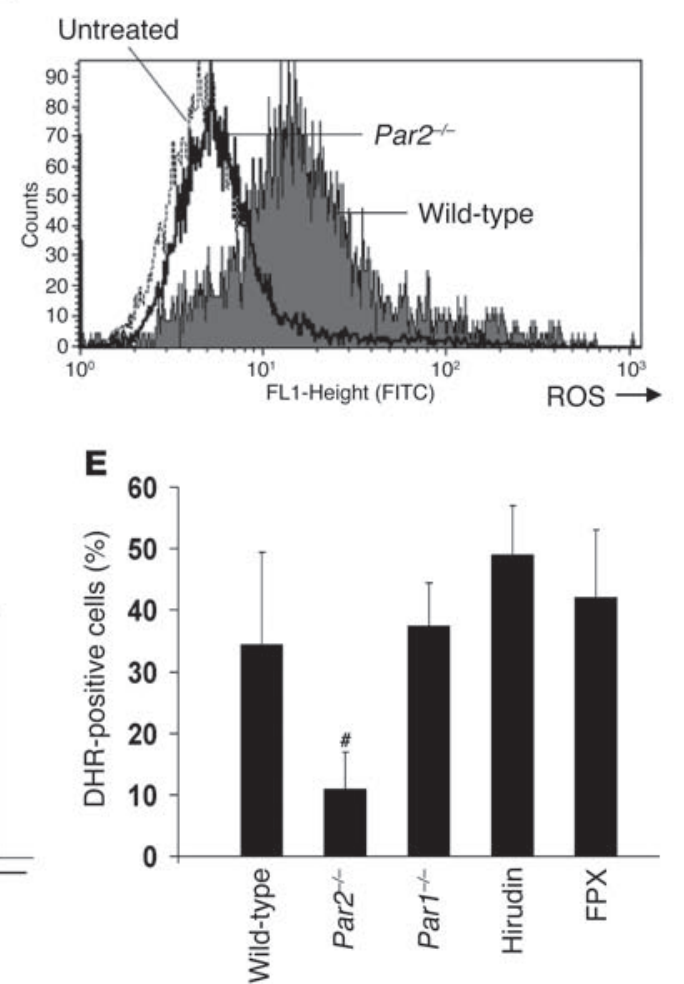

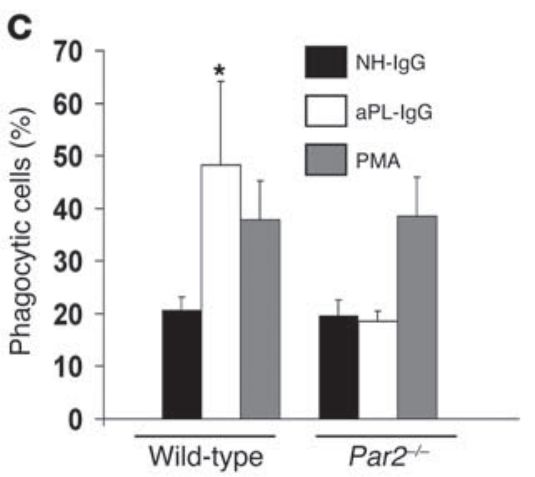

$\mathbf{F}$

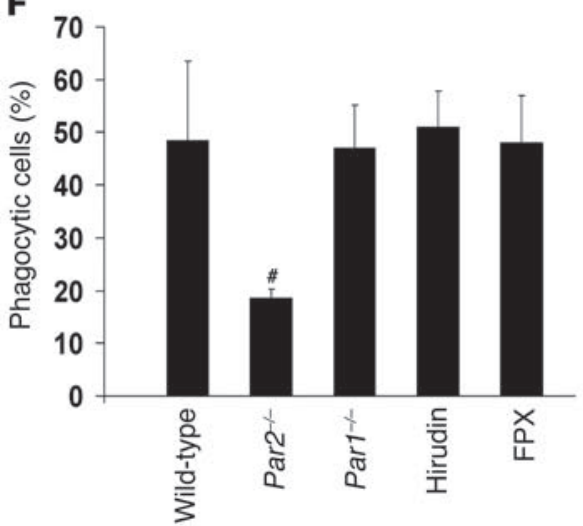

Figure 1

PAR2 is required for aPL-IgG-induced neutrophil activity. (A) Immunohistochemical detection of TF and PAR2 on neutrophils from aPL-IgG- and $\mathrm{NH}$-IgG-treated mice. Original magnification, $\times 400$. (B) ROS production, measured as DHR-positive cells, on whole blood neutrophils from aPL-IgG-treated mice. The number of DHR-positive neutrophils increased in aPL-IgG-treated wild-type mice compared with untreated mice. ROS production in neutrophils did not increase in aPL-IgG-treated Par2 ${ }^{-/-}$mice. (C and D) Phagocytic cells and DHR-positive cells in wild-type and Par2 ${ }^{-1-}$ mice. The percentage of phagocytic (C) and DHR-positive (D) neutrophils increased in aPL-IgG-treated wild-type mice compared with NH-IgG-treated mice. The absence of PAR2 prevented aPL-IgG-induced ROS production and phagocytosis. Incubation of neutrophils from $P$ ar2 $2^{--}$mice with PMA induced increased ROS generation and phagocytosis, which indicates that the capacity of $P a r 2^{-/-}$mice to generate oxidants or in phagocytosis is normal. (E and F) ROS production and phagocytosis in neutrophils from aPL-lgG-treated mice. (E) Neutrophils from $\mathrm{Par1}^{-1-}$ mice treated with aPL-IgG showed increased ROS generation (E) as well as phagocytosis similar to aPL-IgG-treated wild-type mice (F). Increased ROS production was also observed in neutrophils from mice treated with hirudin or FPX in addition to aPL-IgG. $n=5-7$ per group. ${ }^{*} P<0.05$ versus $\mathrm{NH}-\mathrm{IgG}$; $P<0.05$ versus wild type. Data in $\mathbf{C}-\mathbf{F}$ are mean $\pm \mathrm{SD}$.

Statins prevent fetal injury in aPL-IgG-treated mice. Statins are compounds commonly used to reduce the level of cholesterol in the blood. In addition to lipid lowering, statins are postulated to exhibit pleiotropic properties, such as inhibition of inflammation and coagulation (17). It was also shown previously that simvastatin reduced TF expression and activity in blood monocytes in patients with nephritic syndrome (18). Other statins also suppress TF expression in various cell types $(19,20)$. Knowing that TF is a crucial mediator in aPL-induced pregnancy loss and that statins diminish TF expression, we sought to determine whether statins can prevent pregnancy loss in aPL-IgG-treated mice. We first studied the effects of simvastatin on aPL-IgG-induced pregnancy loss. In accordance with our hypothesis, simvastatin prevented fetal loss in aPL-IgG-treated mice (Figure 4, A and B). A statistically significant difference was observed between aPL-IgG treatment alone and treatment with aPL-IgG plus simvastatin (Figure 4A). Multiple fetal resorptions were observed in uteri from aPL-IgG-treated mice (Figure 4B), while viable fetuses and no fetal resorptions - comparable to NH-IgG-treated mice - were observed in the uteri of mice treated with aPL-IgG plus simvastatin (Figure 4B). In aPL-
IgG-treated wild-type mice, extensive C3 deposition in deciduae as well as embryo debris was observed (Figure 4B). In contrast, the decidual tissue from mice treated with aPL-IgG plus simvastatin showed minimal staining for C3 and intact embryo (Figure 4C).

To prevent complement-mediated autologous attack, host tissues express a number of inhibitors $(21,22)$. Decay-accelerating factor (DAF) is a membrane-bound complement inhibitor that protects trophoblasts from complement attack by inhibiting C3 convertase (23). A recent study reported that simvastatin increases DAF expression and prevents complement activation on HUVECs in vitro (24). Given the important role of complement activation in this model of aPL-induced pregnancy loss $(1,11,12)$, these observations raise the question of whether the protective effects of simvastatin observed in aPL-induced pregnancy loss are caused by increased complement inhibition by DAF. We therefore measured DAF expression on trophoblasts incubated with simvastatin and found abundant expression of DAF on trophoblasts that was not diminished by simvastatin (Figure 4D).

Simvastatin downregulates TF and PAR2 expression and diminishes ROS production and phagocytosis in neutrophils. Knowing that the protective 
A

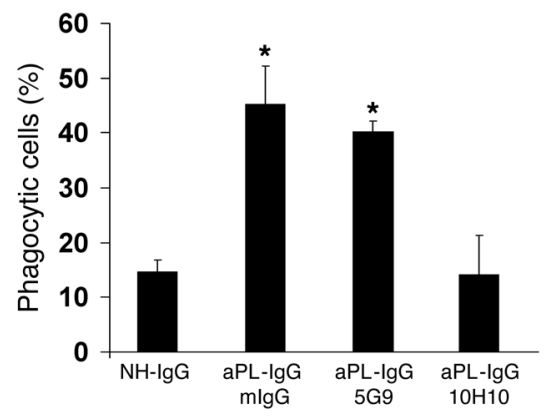

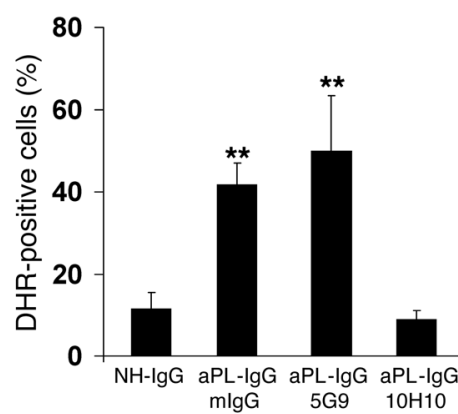

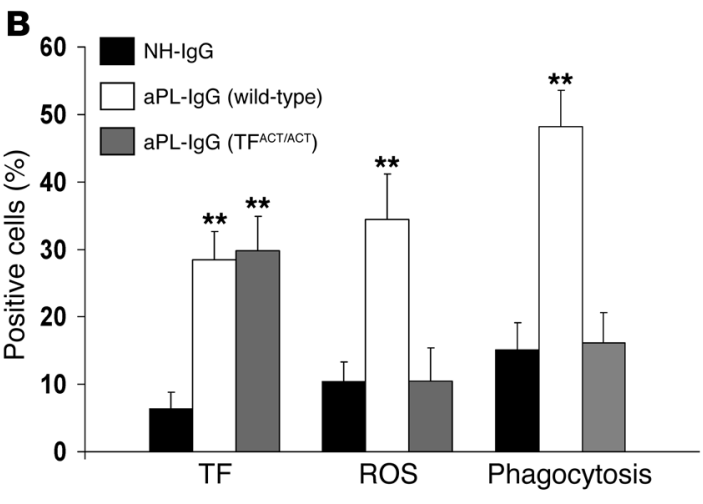

Figure 2

TF/FVIIa/PAR2 signaling is required for aPL-IgG-induced increase in neutrophil activity. (A) Effect of $10 \mathrm{H} 10$ and $5 \mathrm{G} 9$ on neutrophil phagocytosis and neutrophil ROS generation in aPL-IgG-treated mice, as determined by FACS. aPL-IgG-induced phagocytosis and ROS generation were prevented by $10 \mathrm{H} 10$, but not $5 \mathrm{G}$. Mouse IgG1 (mlgG) was used as isotype control. (B) TF expression, ROS production, and phagocytosis in neutrophils from NH-IgG-treated wild-type mice, aPL-IgG-treated wild-type mice, and aPL-IgG-treated TF $\triangle \mathrm{CT} / \triangle \mathrm{CT}$ mice. aPL-IgG-induced ROS production and phagocytosis was not observed in TF ${ }^{\Delta C T / \Delta C T}$ mice. ${ }^{*} P<0.05,{ }^{* \star} P<0.01$ versus NH-lgG. $n=5$ mice per group. All data are mean \pm SD.

effects of simvastatin are not based on increasing anticomplement activity, we hypothesized that treatment with statins would reduce aPL-IgG-induced neutrophil TF and PAR2 expression, diminishing ROS generation and thus preventing trophoblast injury and fetal death. FACS experiments showed that simvastatin prevented TF expression on neutrophils from aPL-IgG-treated mice (aPL-IgG, $28.5 \% \pm 4.5 \%$ TF positive; aPL-IgG plus simvastatin, $10.1 \% \pm 5.7 \%$ TF positive; $P<0.05, n=6-8)$. The increase in PAR2 expression was also reduced by simvastatin (aPL-IgG, $16.2 \% \pm 6.1 \%$ PAR 2 positive; aPL-IgG plus simvastatin, $6.9 \% \pm 2.3 \%$ PAR2 positive; $P<0.01$, $n=5-7)$. Simvastatin treatment had no effect on TF and PAR2 expression in NH-IgG-treated as well as untreated mice (data not shown). Neutrophil activation was also prevented in mice treated with aPL-IgG plus simvastatin; moreover, neutrophils from mice treated with aPL-IgG plus simvastatin showed ROS production that was similar to that in neutrophils from NH-IgG-treated or untreated mice (Figure 5A). The aPL-IgG-dependent increase in neutrophil phagocytosis was also reduced by simvastatin (aPL-IgG, $48.2 \% \pm 11 \%$ of total phagocytic cells; aPL-IgG plus simvastatin, $16.2 \% \pm 2.9 \%$ of total phagocytic cells; $P<0.01, n=5-8)$. Simv-

\section{Figure 3}

$\mathrm{Par2}^{-/-}$mice are protected from trophoblast oxidative injury and fetal death. Pregnant $P a 2^{-1-}$ or wild-type mice were given $10 \mathrm{mg}$ aPL-IgG or NH-IgG i.p. on days 8 and 12. (A) Mice were killed on day 15 of pregnancy, uteri were dissected, and FRF was calculated as described in Methods. In matings of wild-type mice, approximately $40 \%$ of the embryos of mice treated with aPL-IgG were resorbed; in contrast, $P$ ar2 $2^{-/-}$mice showed a reduction in aPL-IgG-induced FRF. ${ }^{*} P<0.05$ versus NH-lgG. $n=5-7$ mice per group. Data are mean \pm SD. (B and $\mathbf{C}$ ) Mice were killed on day 8, $2 \mathrm{~h}$ after aPL-IgG or $\mathrm{NH}$-lgG injection. Uteri were dissected, and decidua sections were cut and stained with an anti-C3 antibody (B) or DHE (C) to measure superoxide generation. (B) The chromogen was DAB (brown), and the counterstain was hematoxylin. In aPL-IgGtreated wild-type mice, there was extensive C3 staining (brown) in deciduae as well as embryo debris (ED). In contrast, the decidual tissue from aPL-IgG-treated Par2 ${ }^{-/-}$mice showed minimal staining for $\mathrm{C} 3$ at the ectoplacental cone (ec) and intact embryo (E). (C) aPL-IgG-induced superoxide production in wild-type mice was attenuated in $\mathrm{Par}^{-/-}$mice. Oxidative damage in deciduae from aPL-IgG-treated PAR2 mice was minimal and not different from that in $\mathrm{NH}-\mathrm{lgG}$-treated wild-type mice. Scale bars: $100 \mu \mathrm{m}$ (B); $40 \mu \mathrm{m}$ (C).
A

B

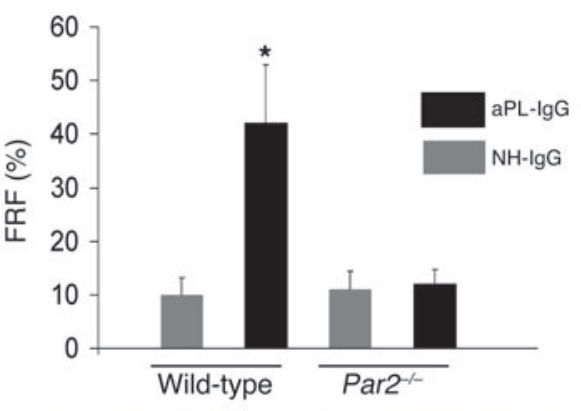

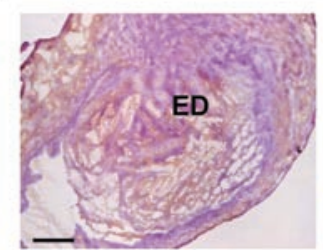

C

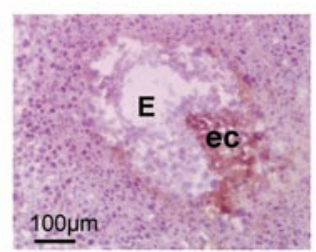

aPL-IgG (Par2 $\left.{ }^{--}\right)$

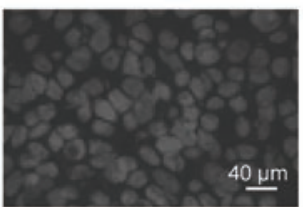

aPL-IgG (wild-type)

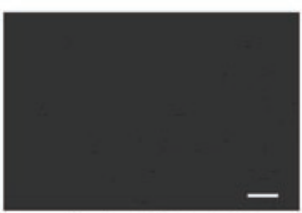

aPL-IgG (Par2-- $)$

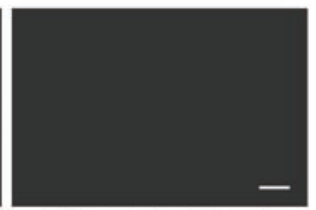

$\mathrm{NH}$-lgG (wild-type) 
A

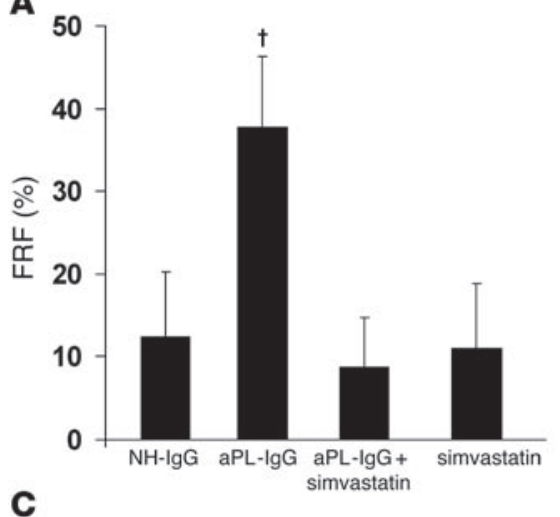

C

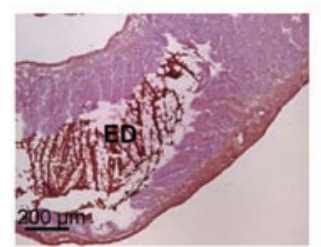

aPL-IgG
B

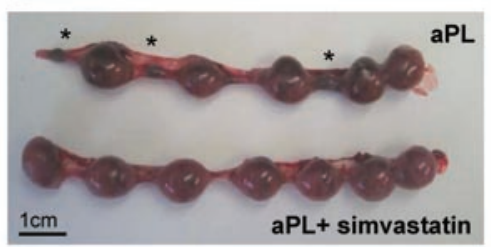

vented pregnancy loss induced by aPL-IgG (Figure $5 \mathrm{E})$. The increased FRF observed in aPL-IgGtreated mice was not present in mice treated with aPL-IgG plus pravastatin (Figure 5E). Pravastatin also downregulated TF and PAR2 expression on neutrophils, which inhibited neutrophil activation (data not shown). These results suggest that statins prevent aPL-induced neutrophil activation, thus protecting trophoblasts from oxidative damage and rescuing the fetuses.

\section{Discussion}

In this study we demonstrated that TF-dependent signaling in neutrophils was responsible for fetal damage in a mouse model of APS. Our results showed that aPL-IgG-induced TF expression on neutrophils and formation of a TF/FVIIa complex contributed to oxidative burst, trophoblast injury, and fetal death by signaling through PAR2. Furthermore, we showed that statins reduced the expression of TF and PAR2 on neutrophils and prevented aPL-IgG-induced fetal loss.

Agonists of PARs, notably PAR2, induce inflammation in many tissues (28). The association of PAR2 with TF-dependent signaling pathways has become increasingly apparent in many inflammatory diseases (29). We previously reported increased TF expression on neutrophils from aPL-treated mice (1). A major concern with regard to the increased TF expression observed in these mice is the origin of TF. The question of whether neutrophils are capable of synthesizing TF is the subject of extensive debate $(30,31)$. Our RT-PCR experiments clearly demonstrate that mouse neutrophils from abortion-prone aPL-IgG-treated mice expressed TF. Østerud previously showed that human neutrophils can acquire circulating TF from activated monocytes (31). We not only found TF mRNA in mouse neutrophils from aPL-IgGtreated mice, but we also found TF-positive neutrophils in aPL-IgG-treated mice that were depleted of monocytes (data not shown). Based on

astatin inhibited aPL-IgG-induced TF and PAR2 mRNA expression (Figure 5, B and C). aPL-IgG-dependent increases in free radical-mediated lipid peroxidation in decidual tissue were also ameliorated by simvastatin treatment (Figure 5D). The increased superoxide production observed in deciduae from aPL-IgG-treated mice was not present when mice received simvastatin treatment (Figure 5D). Free radical-mediated lipid peroxidation in deciduae from mice treated with aPL-IgG plus simvastatin was comparable to deciduae from NH-IgG-treated mice (Figure 5D). By inhibiting the synthesis and expression of TF and PAR2 induced by aPL-IgG, simvastatin prevented neutrophil activation, placental oxidative damage, and fetal death.

In an epidemiologic study of adverse birth outcomes following gestational exposure to statins, pravastatin was shown to be the safest drug (25). Because pravastatin is minimally present in the embryo and diminishes leukocyte activity $(26,27)$, we decided to test the effects of pravastatin in our model of aPL-induced pregnancy loss. Similar to the effects of simvastatin, pravastatin pre- these results, we conclude that mouse neutrophils from aPL-IgGtreated mice express endogenously synthesized TF. Our results are in accordance with Ritis et al., who showed that human neutrophils synthesize TF when incubated with C5a (32). We previously showed that TF expression on mouse neutrophils is dependent on an interaction between C5a and C5aR (1). We propose that C5a may be the trigger for TF expression on neutrophils in our model of aPL-induced fetal injury.

Neutrophils are important effectors of fetal injury in aPLinduced pregnancy loss and are also known to express various PARs (33). It was reported that PAR2 agonists upregulate cell adhesion molecule expression and cytokine production by human neutrophils (33). Of note, a significant increase of PAR2 expression was observed on the cell surface of neutrophils from septic patients compared with healthy volunteers (34), which indicates that PAR2 may be involved in the pathophysiology of neutrophil activation observed during sepsis in humans. In our present study, similar to the results of these prior studies in sepsis, we found 
A

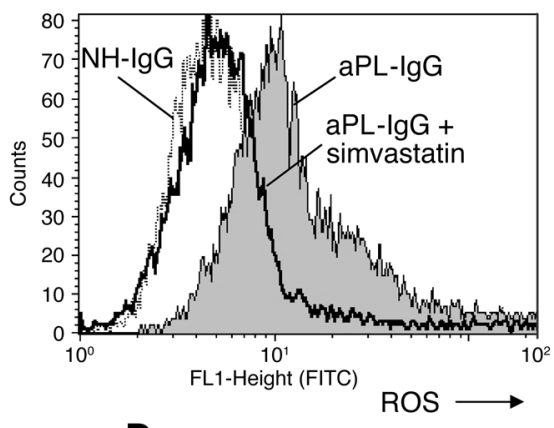

D
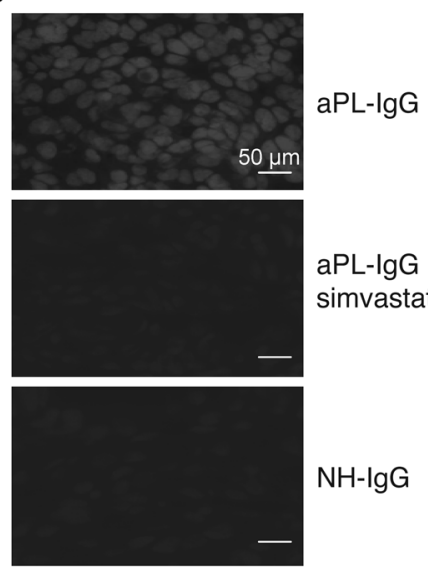

aPL-lgG + simvastatin

$\mathrm{NH}-\lg \mathrm{G}$
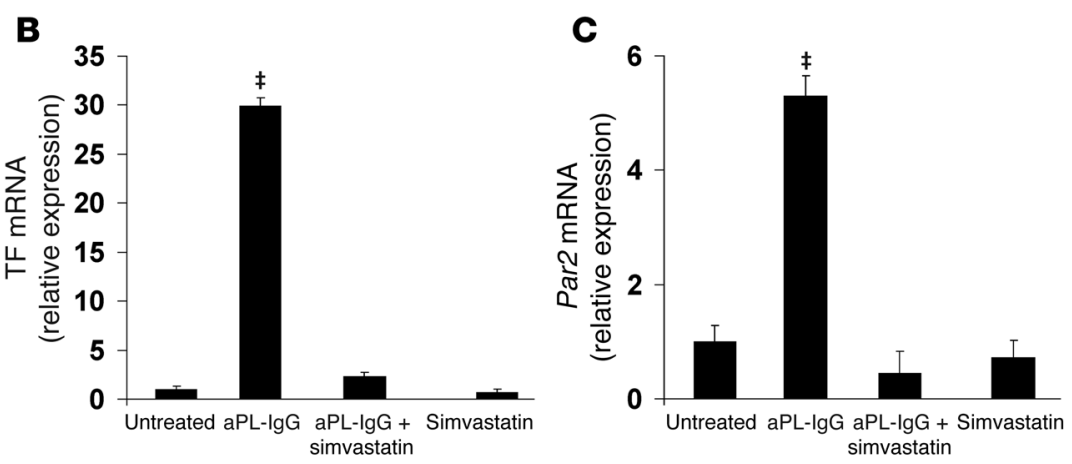

E

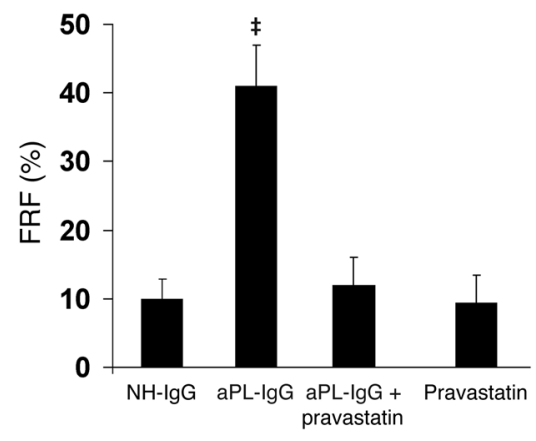

Figure 5

Statins inhibit TF and PAR2 synthesis and prevent neutrophil oxidative burst and oxidative damage in aPL-IgG-treated mouse placentas. Pregnant C57BL/6 mice were given aPL-lgG or NH-lgG as in Figure 3; some also received $20 \mu \mathrm{g}$ simvastatin or $5 \mu \mathrm{g}$ pravastatin i.p. $18 \mathrm{~h}$ before aPL-IgG administration ( $n=5-10$ mice per group). (A) On day 8, $2 \mathrm{~h}$ after aPL-IgG or NH-IgG injection, a blood sample was drawn in order to determine ROS production and phagocytosis in neutrophils by FACS, measured as the number of DHR-positive cells. aPL-IgG increased ROS production, whereas simvastatin prevented neutrophil oxidative burst in aPL-lgG-treated mice. The number of DHR-positive neutrophils was similar between NH-IgG treated and aPL-IgG plus simvastatin-treated mice. (B and C) RT-PCR analysis was performed in isolated neutrophils to quantify TF and PAR2 gene expression. Neutrophils from aPL-lgG-treated mice showed a 28-fold increase in TF mRNA (B) and a 5-fold increase in Par2 mRNA (C). Simvastatin prevented aPL-IgG-induced increase in TF and PAR2 synthesis. (D) Mice were killed on day 8 , and deciduae were removed to determine superoxide generation using DHE fluorescence. Increased free radical-mediated lipid peroxidation was observed in deciduae from aPL-IgG-treated mice. No oxidative damage was observed in NH-IgG and aPL-IgG plus simvastatin-treated mice. Scale bars: $50 \mu \mathrm{m}$. (E) Mice were killed on day 15 of pregnancy, uteri were dissected, and FRF was calculated. Similar to the effects of simvastatin, pravastatin treatment prevented fetal loss. ${ }^{\ddagger} P<0.05$ versus aPL-IgG plus simvastatin or pravastatin as appropriate.

increased synthesis and expression of PAR2 in neutrophils from aPL-IgG-treated mice. Experiments performed in Par2 ${ }^{-/}$mice demonstrated that PAR2 signaling was required for TF-induced neutrophil activation, trophoblast injury, and pregnancy loss in aPL-IgG-treated mice. The finding that PAR2 deficiency decreases phagocytosis in neutrophils was also reported in murine pulmonary pseudomonal infection (35). However, the decreased neutrophil phagocytosis observed in this model was associated with reduced bacterial clearance and increased lung inflammation (35). In our present study, decreased neutrophil activity observed in the absence of PAR2 prevented oxidative injury to trophoblasts and rescued pregnancy in APS.

Studies performed in Par2 ${ }^{-/-}$mice identified PAR2 as the receptor that interacts with TF to induce proinflammatory effects in neutrophils. While the lack of PAR2 prevented neutrophil activation and protected pregnancy in APS, the absence of PAR1 did not prevent neutrophil activation in aPL-IgG-treated mice. These data indicate that PAR1 is not required for aPL-induced fetal loss. Our data also indicated that neither generation of thrombin and FXa nor PAR1 activation are required for increased neutrophil activity, suggesting that the TF/FVIIa complex is the primary activator of PAR2 on neutrophils. Several studies analyzing the role of TF in growth of human tumor cells in mice have used the anti-hTF antibodies $10 \mathrm{H} 10$ and $5 \mathrm{G} 9(14,36,37)$, indicating that these antibodies are valuable tools to distinguish the role of TF in coagulation versus signaling. In the present study, treatment with $10 \mathrm{H} 10$, which selectively blocks TF/FVIIa signaling through PAR2, prevented neutrophil activation induced by aPL-IgG, confirming the role of PAR2 in fetal demise in APS. The lack of protection observed with 5G9, which blocks TF-induced coagulation, was consistent with the previous observation that anticoagulation with hirudin or FPX does not prevent neutrophil activation and does not prevent miscarriages induced by aPL (13). The importance of TF-dependent signaling pathways in aPL-induced fetal injury was further substantiated by studies performed in $\mathrm{TF}^{\Delta \mathrm{CT} / \Delta \mathrm{CT}}$ mice, which cannot induce cellular effects 
in response to TF/FVIIa. The protection from oxidative damage observed in $\mathrm{TF}^{\Delta \mathrm{CT} / \Delta \mathrm{CT}}$ mice suggests that TF/FVIIa/PAR2 signaling is required for aPL-induced neutrophil activity and pregnancy complications. The absence of the cytoplasmic domain of TF was previously associated with a diminished inflammatory response in a murine arthritis model (38). However, studies performed in a mouse model of oxygen-induced retinopathy showed that loss of the TF cytoplasmic domain resulted in increased angiogenesis mediated by TF/FVIIa signaling through PAR2 (39).

Our findings do not exclude a contribution of PAR2 in placental endothelial cells and trophoblasts to aPL-induced fetal death. PAR2 is found in mouse trophoblasts and has been associated with reduced proliferation and abnormal placentation $(40,41)$. Therefore, TF/FVIIa/PAR2 signaling on trophoblasts may also contribute to trophoblast injury and pregnancy loss. However, human and mouse trophoblast cell lines incubated with aPL-IgG did not show any increase in PAR2 expression (data not shown). These results, together with our previous finding that TF on neutrophils is a crucial mediator of aPL-induced trophoblast damage in aPL-treated mice (1), support the role of PAR2 expression on neutrophils in the pathogenesis of abortions in APS.

Statins are pluripotent agents exhibiting multiple non-lipidlowering actions (17). Besides their established role in the management of hypercholesterolemia, statins may also have beneficial actions in other pathological conditions, including (but not limited to) cancer, solid organ transplantation, cerebrovascular ischemic events, renal diseases, rheumatoid arthritis, autoimmune diseases, and sepsis (42). Statins reduce TF synthesis, expression, and activity in many different cells, such as blood monocytes, endothelial cells, and breast carcinoma cells $(18,19,42)$. In our present study, simvastatin and pravastatin decreased TF and PAR2 expression on neutrophils and prevented pregnancy loss induced by aPL-IgG. It has previously been noted that statins abolish the antiapoptotic signaling of TF/FVIIa in breast cancer cells (43); this effect may also be caused by PAR2 downregulation. Here, statins prevented neutrophil activation by downregulating TF and PAR2 expression and protected fetuses from aPL-IgG-induced injury. In a similar way, Broz et al. found that statins diminish phagocytosis in macrophages in atherosclerotic plaques (44). Statins also attenuated aPL-IgG-induced inflammation and complement deposition induced by aPL-IgG. By preventing neutrophil activation, statins might inhibit complement $\mathrm{C} 3$ and properdin release. Properdin and C3 secretion by neutrophils creates a complement alternative pathway amplification loop perpetuating complement activation on placental tissue (12).

Our previous work demonstrated the benefits of heparin in aPL-induced pregnancy loss in mice (13). This raises the question of whether statins would provide benefits greater than those of heparin for the treatment of patients with aPL. Heparin prevents fetal loss by blocking the activation of complement induced by aPL, rather than via their anticoagulant properties (13). However, heparin has a number of side effects. Women on long-term administration of heparin are at higher risk of bleeding and should be monitored for the possible development of osteoporosis and spontaneous fracture. On the other hand, statins show a very low incidence of serious side effects and can be conveniently administered orally. We believe that by targeting the downstream effects of activation of the complement pathway at the level of TF with statins, we decrease the pathological TF expression and subsequent inflammation without markedly impacting hemostasis.
To date, epidemiological data collected by other investigators suggest that statins are not major teratogens. Pregnancy outcomes after maternal exposure to statins showed no evidence of increased congenital abnormalities (45). Moreover, recent studies identified new benefits of statins in pregnancy in mice. It was reported that the use of statins in pregnant mice consuming a high-fat, highcholesterol diet leads to reduced cardiovascular risk factors in offspring that are sustained into adulthood (46).

Lipophilic statins reaching the embryo may downregulate biosynthesis of cholesterol as well as many important metabolic intermediates and may have secondary effects (25). Pravastatin is a hydrophilic statin, and minimal amounts are found in the embryo after exposure (25), which suggests that pravastatin would be helpful to treat pregnancy complications. In addition, pravastatin is one of a group of statins with no effect on mitochondrial activity, while other statins can cause mitochondrial toxicity by inhibiting oxidative phosphorylation (47). It was also reported that statins may have beneficial effects in other pregnancy complications such as eclampsia (48). Statins have been shown to decrease release of the antiangiogenic factor soluble Flt-1 (sFlt-1) from endothelial cells and normal-term placental villous explants (48); thus, statins may alleviate the symptoms of preeclampsia.

We recognize that drugs should be used during pregnancy only if the benefits outweigh the risks. Given the pleiotropic effects of statins related to many pathophysiological determinants of pregnancy loss in APS and considering the beneficial effects of statins in our animal studies, we postulate that statins may be a good treatment for women with aPL-induced pregnancy complications. Moreover, preventing neutrophil activation by inhibiting TF/FVIIa/PAR2 signaling with statins may have important implications in many other inflammatory diseases.

\section{Methods}

\section{Transgenic mice}

All protocols were approved by the Institutional Animal Care and Use Committee of Hospital for Special Surgery and performed in compliance with institutional guidelines. HCV mice, which express normal levels of

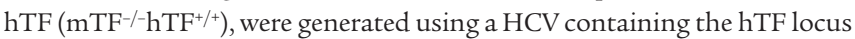
(15). TNF- $\alpha$-deficient, $\mathrm{Par}^{-/-}$, and C57BL/6 mice were purchased from The Jackson Laboratory. Par1 $1^{-/-}$mice were kindly provided by S. Coughlin (Cardiovascular Research Institute, UCSF, San Francisco, California, USA; ref. 49). $\mathrm{TF}^{\Delta \mathrm{CT} / \triangle \mathrm{CT}}$ mice, which lack the 18 carboxyterminal amino acids of the cytoplasmic domain of TF, were generated by the Cre-lox recombination technique (50).

\section{Preparation of antibodies for in vivo studies}

aPL-IgG was obtained from patients with APS (characterized by high-titer aPL, i.e., >140 GPL units; thromboses; and/or pregnancy losses). NH-IgG was obtained from healthy nonautoimmune individuals. Individuals provided informed consent before participating in these studies. All protocols were approved by the clinical research review board of Hospital for Special Surgery and performed in compliance with institutional guidelines.

Anti-TF mAbs 5G9 and 10H10 are of the IgG1 subclass and selective inhibitors of TF-dependent coagulation and TF/FVIIa signaling through PAR2, respectively (51). Antibody 5G9 binds to TF and the preformed TF/FVIIa complex, inhibiting the formation of the TF/FVIIa/FXa ternary initiation complex of coagulation (52). Antibody $10 \mathrm{H} 10$ binds to a partially overlapping epitope on hTF, but does not block coagulation because it does not inhibit binding of FVIIa or the function of the TF/ 
FVIIa complex $(53,54)$. For all cellular and in vivo studies, antibodies were purified under endotoxin-free conditions (16). Mouse IgG1 was used as isotype control antibody.

\section{Murine aPL-induced fetal loss model}

Adult mice (6-8 weeks) were used in all experiments. On days 8 and 12 of pregnancy, females were treated with injection of $10 \mathrm{mg}$ aPL-IgG or NH-IgG i.p. To block TF, HCV mice were treated with i.p. injections of $0.5 \mathrm{mg}$ $10 \mathrm{H} 10,0.5 \mathrm{mg} 5 \mathrm{G} 9$ (16), or mouse IgG1 on days 6 and 10 of pregnancy. To study the role of statins on aPL-induced pregnancy loss, mice were treated with $20 \mu \mathrm{g}$ simvastatin (Sigma-Aldrich) or $5 \mu \mathrm{g}$ pravastatin (Sigma-Aldrich) i.p. $18 \mathrm{~h}$ prior to aPL-IgG injections on days 6 and 10. A group of mice was treated with the thrombin direct inhibitor hirudin $(8 \mu \mathrm{g} /$ twice daily s.c.; Sigma-Aldrich) or the specific FXa inhibitor FPX sodium (Arixtra, $10 \mu \mathrm{g} /$ day s.c.; Sanofi-Synthelabo LLC) from day 7 to day 14 of pregnancy. On day 8, 2 h after aPL-IgG or NH-IgG injection, a sample of blood was drawn in order to measure oxidative burst, phagocytosis, and TF and PAR2 expression and synthesis in neutrophils. FPX and hirudin exhibited the expected anticoagulant activities - elevated partial thromboplastin time (untreated, $29 \pm 2 \mathrm{~s}$; hirudin, $69 \pm 5 \mathrm{~s}$ ) and increased FXa inhibitory activity (FPX, 91\% $\pm 5 \%$ inhibition) - at the time the blood was drawn to perform FACS experiments. On day 15 of pregnancy, mice were euthanized, their uteri were dissected, and FRF was calculated as the number of resorptions per the total number of formed fetuses and resorptions. Resorption sites are easily identified and result from loss of a previously viable fetus. For immunohistochemistry studies, deciduae were removed from mice on day 8 of pregnancy, 2 h after treatment with aPL-IgG.

Simvastatin (Sigma-Aldrich) was prepared as a $4 \mathrm{mg} / \mathrm{ml}$ stock. Briefly, $4 \mathrm{mg}$ simvastatin was dissolved in $100 \mu \mathrm{l}$ ethanol and $150 \mu \mathrm{l} 0.1 \mathrm{~N} \mathrm{NaOH}$ and incubated at $50^{\circ} \mathrm{C}$ for $2 \mathrm{~h}$, after which the $\mathrm{pH}$ was adjusted to 7 and the total volume was corrected to $1 \mathrm{ml}$. The stock solution was diluted to the appropriate concentration in sterile PBS. Pravastatin (Sigma-Aldrich) was directly dissolved in sterile PBS.

\section{Immunohistochemistry and immunocytochemistry}

For immunohistochemistry studies, deciduae from day 8 of pregnancy were frozen in OCT compound and cut into $10-\mu \mathrm{m}$ sections. Sections were stained for neutrophils using rat anti-mouse granulocyte RB6-8C5 mAb (BD Biosciences - Pharmingen) and C3 with goat anti-mouse C3 (Cappel ICN). A HRP-labeled secondary antibody and DAB as substrate were used to develop the reaction.

\section{FACS analysis of mouse neutrophils}

Peripheral blood was used for all FACS experiments. Heparinized venous blood was drawn from the submandibular vein by the cheek-pouch method and processed immediately for FACS analysis.

TF and PAR2 expression on mouse neutrophils. To study the presence of TF and PAR2 on peripheral blood neutrophils, 2-color FACS analysis was performed. To measure TF expression, heparinized whole mouse blood was stained with FITC-labeled anti-mouse Ly-6G (Gr-1) for fluorescence channel 1 (FL1) (BD Biosciences - Pharmingen) to identify neutrophils and biotinylated rat anti-mTF antibody $1 \mathrm{H} 1$ (kindly provided by D. Kirchhofer, Genetech, San Francisco, California, USA) and streptavidin-PerCP (BD Biosciences - Pharmingen) as the fluorescence channel 3 (FL3) fluorochrome. To detect PAR2 expression, biotinylated mouse anti-mouse PAR2 $\mathrm{mAb}$ (Santa Cruz Biotechnology Inc.) was used. Red cells were lysed with ACK buffer. Preparations were then incubated with SA-PerCP and analyzed by FACS using FACscan (BD Biosciences).

Respiratory burst activity in neutrophils. Intracellular ROS production was assessed with dihydrorhodamine 123 (DHR; Sigma-Aldrich) by flow cytometry. This primarily nonfluorescent dye becomes fluorescent upon oxidation to rhodamine by ROS produced during the respiratory burst. DHR $(10 \mu \mathrm{mol} / \mathrm{l})$ was added to heparinized whole blood, and this mixture was incubated at $37^{\circ} \mathrm{C}$ for $45 \mathrm{~min}$. Red cells were lysed as described above, and ROS production was analyzed by absorbance in FL1.

Phagocytosis assay. Whole blood was incubated for $1 \mathrm{~h}$ at $37^{\circ} \mathrm{C}$ and $5 \%$ $\mathrm{CO}_{2}$ with fluorescent yellow-green latex beads (1:25 phagocyte/target ratio; Sigma-Aldrich) that had been preincubated with PBS plus 5\% BSA for $1 \mathrm{~h}$ at $37^{\circ} \mathrm{C}$. Internalization of beads by neutrophils was determined by flow cytometry using a FACSCalibur (BD Biosciences) flow cytometer.

\section{Assessment of superoxide production in decidual tissue by dibydroethidium fluorescence}

In situ superoxide levels were assessed using the fluorescent probe dihydroethidium (DHE). Deciduae from day 8 of pregnancy were frozen in OCT compound, cut into $10-\mu \mathrm{m}$ sections, and incubated with $10 \mu \mathrm{mol} / 1$ DHE for $30 \mathrm{~min}$ at $37^{\circ} \mathrm{C}$. Subsequently, the sections were washed, and fluorescence images were obtained ( $520 \mathrm{~nm}$ excitation; $605 \mathrm{~nm}$ emission). To exclude an influence of the embedding procedure on fluorescence, samples from all treatment groups were embedded in the same block and analyzed simultaneously.

\section{FACS analysis of DAF}

Cell surface expression of DAF was measured by flow cytometry on untreated and statin-treated trophoblast-like BeWo cells. BeWo cells were incubated with $10 \mu \mathrm{g} / \mathrm{ml}$ simvastatin or $5 \mu \mathrm{g} / \mathrm{ml}$ pravastatin. At $18 \mathrm{~h}$ after incubation, DAF expression on the surface of BeWo cells was quantified by flow cytometry using a specific antibody (anti-human CD55; eBioscience).

\section{TF and PAR2 $m$ RNA expression analysis}

Heparinized mouse blood, diluted with Hanks buffered salt solution, was layered onto discontinuous Ficoll/Hypaque gradients (Pharmacia Fine Chemicals) in $12-\mathrm{mm} \times 75-\mathrm{mm}$ test tubes. The gradients, 1 tube per mouse, were centrifuged at $800-1,000 \mathrm{~g}$ for 15 minutes, and the lower interface of cells, containing neutrophils and red blood cells, was harvested. The red blood cells were removed by hypotonic lysis, and the neutrophils were counted, lysed in the recommended buffer for RNA purification, and stored at $-70^{\circ} \mathrm{C}$. For real-time PCR, total RNA from neutrophils was extracted using a RNeasy Mini kit (Qiagen), and $1 \mu \mathrm{g}$ total RNA was reverse transcribed using a First Strand cDNA Synthesis kit (Fermentas Life Sciences). Relative quantification of gene expression was performed by real-time PCR using iQ SYBR-Green Supermix on the iCycler iQ thermal cycler (BioRad) following the manufacturer's protocols. Primer sequences were as follows: mouse GAPDH sense, 5'-TTCACCACCATGGAGAAGGC-3'; antisense, 5'-GGCATGGACTGTGGTCATGA-3'; mTF sense, 5' -AGCTACTGCTTTTTTGTACAAGCTATG-3'; antisense, 5' TGTACCGTTTCGTTCGTCCTAA-3'; mouse PAR2 sense, $5^{\prime}$-TGGCCATTGGAGTCTTCCTGTT-3'; antisense, 5'-TAGCCCTCTGCCTTTTCTTCTC$3^{\prime}$. Relative expression was normalized to GAPDH levels. The generation of only the correct size amplification products was confirmed using agarose gel electrophoresis.

\section{Statistics}

Data are expressed as mean $\pm \mathrm{SD}$. After confirming that the data were normally distributed (Kolomogorov-Smirnov test of normalcy), statistical analyses were conducted using 2-tailed Student's $t$ test to compare differences in means. Data were processed using the SigmaStat (version 3.1; Systat) statistical program for Windows. A $P$ value less than 0.05 was considered to be statistically significant. 


\section{Acknowledgments}

We thank Shaun Coughlin for making $\mathrm{Par1}^{-/-}$mice available for our studies. We are grateful to Daniel Kirchhofer for generously providing $1 \mathrm{H} 1$ anti-mTF antibody. This research was supported by grants from the Mary Kirkland Center for Lupus Research at Hospital for Special Surgery, Weill Medical College of Cornell University (to G. Girardi), and the NIH (to N. Mackman).
Received for publication May 1, 2008, and accepted in revised form July 16, 2008.

Address correspondence to: Guillermina Girardi, Hospital for Special Surgery, Caspary Research Building, Room 302, Weill Medical College, Cornell University, 535 E. 70th St., New York, New York 10021, USA. Phone: (212) 774-2187; Fax: (212) 717-1192; E-mail: girardig@hss.edu.
1. Redecha, P., et al. 2007. Tissue factor: a link between $\mathrm{C} 5 \mathrm{a}$ and neutrophil activation in antiphospholipid antibody induced fetal injury. Blood. 110:2423-2431.

2. Monroe, D.M., and Key, N.S. 2007. The tissue factor-factor VIIa complex: procoagulant activity, regulation, and multitasking. J. Thromb. Haemost. 5:1097-1105.

3. Strukova, S. 1006. Blood coagulation-dependent inflammation. Coagulation-dependent inflammation and inflammation-dependent thrombosis. Front. Biosci. 11:59-80.

4. Camerer, E., Huang, W., Coughlin, S.R. 2000 Tissue factor- and factor $\mathrm{X}$-dependent activation of protease-activated receptor 2 by factor VIIa. Proc. Natl. Acad. Sci. U. S. A. 97:5255-5260.

5. Ruf, W., Dorfleutner, A., and Riewald, M. 2003. Specificity of coagulation factor signaling. J. Thromb. Haemost. 1:1495-1503.

6. Lindner, J.R., et al. 2000. Delayed onset of inflammation in protease-activated receptor-2-deficient mice. J. Immunol. 165:6504-6510.

7. Zimmerman, B.J., et al. 1994. Thrombin receptor peptide-mediated leukocyte rolling in rat mesenteric venules: roles of P-selectin and sialyl Lewis X. Am. J. Physiol. 267:H1049-H1053.

8. Erlich, J.H., et al. 2000. Inhibition of the tissue factor-thrombin pathway limits infarct size after myocardial ischemia-reperfusion injury by reducing inflammation. Am. J. Pathol. 157:1849-1862.

9. Sevastos, J., et al. 2007. Tissue factor deficiency and PAR-1 deficiency are protective against renal ischemia reperfusion injury. Blood. 109:577-583.

10. Stone, S., et al. 2006. The placental bed in pregnancies complicated by primary antiphospholipid syndrome. Placenta. 27:457-467.

11. Holers, V.M., et al. 2003. Complement C3 activation is required for antiphospholipid antibodyinduced fetal loss. J. Exp. Med. 195:211-220.

12. Girardi, G., et al. 2004. Complement C5a receptors and neutrophils mediate fetal injury in the antiphospholipid syndrome. J. Clin. Invest. 112:1644-1654.

13. Girardi, G., Redecha, P., and Salmon, J.E. 2004. Heparin prevents antiphospholipid antibody-induced fetal loss by inhibiting complement activation. Nat. Med. 10:1222-1226.

14. Versteeg, H.H. 2008. Inhibition of tissue factor signaling suppresses tumor growth. Blood. 111:190-199.

15. Pawlinski, R., et al. 2007. Role of cardiac myocyte tissue factor in heart hemostasis. J. Thromb. Haemost. 5:1693-1700.

16. Ahamed, J., et al. 2007. Regulation of macrophage procoagulant responses by the tissue factor cytoplasmic domain in endotoxemia. Blood. 109:5251-5259.

17. Takemoto, M., and Liao, J.K. 2001. Pleiotropic effects of 3-hydroxy-3-methylglutaryl coenzyme A reductase inhibitors. Arterioscler. Thromb. Vasc. Biol. 21:1712-1719.

18. Wei, J.L., Cui, H.M., and Ma, C.Y. 2007. Simvastatin inhibits tissue factor and plasminogen activator inhibitor-1 secretion by peripheral blood mononuclear cells in patients with primary nephrotic syndrome. Eur. J. Med. Res. 12:216-221.
19. Pierangeli, S.S., and Ferrara, D.E. 2005. More on: fluvastatin inhibits up-regulation of tissue factor expression by antiphospholipid antibodies on endothelial cells. J. Thromb. Haemost. 3:1112-1113.

20. Kunieda, Y. et al. 2003. HMG CoA reductase inhibitor suppresses the expression of tissue factor and plasminogen activator inhibitor- 1 induced by angiotensin II in cultured rat aortic endothelial cells. Thromb. Res. 110:227-234.

21. Volanakis, J.E., and Frank, M. 1998. The buman complement system in health and disease. Dekker. New York, New York, USA. 672 pp.

22. Lambris, J.D. 1990. The third component of complement: chemistry and biology. Springer Verlag. Berlin, Germany. 251 pp.

23. Sun, X., et al. 1999. Role of decay-accelerating factor in regulating complement activation on the erythrocyte surface as revealed by gene targeting. Proc. Natl. Acad. Sci. U. S. A. 96:628-633.

24. Mason, J.C., et al. 2002. Statin-induced expression of decay-accelerating factor protects vascular endothelium against complement-mediated injury. Circ. Res. 91:696-703.

25. Edison, R.J., and Muenke, M. 2005. Mechanistic and epidemiologic considerations in the evaluation of adverse birth outcomes following gestational exposure to statins. Am. J. Med. Genet. A. 135:230-231.

26. Rosenson, R., and Tangney, C. 1998. Antiatherosclerotic properties of statins. JAMA. 279:1643-1650.

27. Kubes, P., Suzuki, M., and Granger, D.N. 1991 Nitric oxide: an endogenous modulator of leukocyte adhesion. Proc. Natl. Acad. Sci. U. S. A. 88:4651-4655.

28. Bunnett, N.W. 2006. Protease-activated receptors: how proteases signal to cells to cause inflammation and pain. Semin. Thromb. Hemost. 32:39-48.

29. Versteeg, H.H., and Ruf, W. 2006. Emerging insights in tissue factor-dependent signaling events. Semin. Thromb. Hemost. 32:24-32.

30. Nakamura, S., Imamura, T., and Okamoto, K. 2004. Tissue factor in neutrophils: yes. J. Thromb. Haemost. 2:214-217.

31. Østerud, B. 2004. Tissue factor in neutrophils: no. J. Thromb. Haemost. 2:218-220.

32. Ritis, K., et al. 2006. A novel C5a receptor-tissue factor cross-talk in neutrophils links innate immunity to coagulation pathways. J. Immunol. 177:4794-4802.

33. Howells, G.L., et al. 1997. Proteinase-activated receptor-2: expression by human neutrophils. J. Cell Sci. 110:881-887.

34. Shpacovitch, V.M., et al. 2007. Agonists of proteinase-activated receptor-2 affect transendothelial migration and apoptosis of human neutrophils. Exp. Dermatol. 16:799-806.

35. Moraes, T.J., et al. 2008. Role of PAR2 in murine pulmonary pseudomonal infection. Am. J. Physiol. Lung Cell Mol. Physiol. 294:L368-L377.

36. Fischer, E.G., Ruf, W., and Mueller, B.M. 1995. Tissue factor-initiated thrombin generation activates the signaling thrombin receptor on malignant melanoma cells. Cancer Res. 55:1629-1632.

37. Mueller, B.M., Reisfeld, R.A., Edgington, T.S., and Ruf, W. 1992. Expression of tissue factor by melanoma cells promotes efficient hematogenous metastasis. Proc. Natl. Acad. Sci. U. S. A. 89:11832-11836.
38. Yang, Y.H., et al. 2004. Reduction in arthritis severity and modulation of immune function in tissue factor cytoplasmic domain mutant mice. Am. J. Pathol. 164:109-117.

39. Uusitalo-Jarvinen, H., et al. 2007. Role of protease activated receptor 1 and 2 signaling in hypoxiainduced angiogenesis. Arterioscler. Thromb. Vasc. Biol. 27:1456-1462.

40. O'Brien, P.J. 2003. Thrombin receptors and proteaseactivated receptor- 2 in human placentation: receptor activation mediates extravillous trophoblast invasion in vitro. Am. J. Pathol. 163:1245-1254.

41. Isermann, B., et al. 2003. The thrombomodulinprotein C system is essential for the maintenance of pregnancy. Nat. Med. 9:331-337.

42. Paraskevas, K.I., Tzovaras, A.A., Briana, D.D., and Mikhailidis, D.P. 2007. Emerging indications for statins: a pluripotent family of agents with several potential applications. Curr. Pharm. Des. 13:3622-3636.

43. Aberg, M., Wickström, M., and Siegbahn, A. 2008. Simvastatin induces apoptosis in human breast cancer cells in a NFkappaB-dependent manner and abolishes the anti-apoptotic signaling of TF/FVIIa and TF/FVIIa/FXa. Thromb. Res. 122:191-202.

44. Broz, P., et al. 2008. Inhibition of macrophage phagocytotic activity by a receptor-targeted polymer vesicle-based drug delivery formulation of pravastatin. J. Cardiovasc. Pharmacol. 51:246-252.

45. Pollack, P.S., et al. 2005. Pregnancy outcomes after maternal exposure to simvastatin and lovastatin. Birth Defects Res. A Clin. Mol. Teratol. 73:888-896.

46. Elahi, M.M., et al. 2008. Statin treatment in hypercholesterolemic pregnant mice reduces cardiovascular risk factors in their offspring. Hypertension. 51:939-944.

47. Wagner, B.K., et al. 2008. Large-scale chemical dissection of mitochondrial function. Nat Biotechnol. 26:343-351.

48. Cudmore, M., et al. 2007. Negative regulation of soluble Flt-1 and soluble endoglin release by heme oxygenase-1. Circulation. 115:1789-1797.

49. Connolly, A.J., Suh, D.Y., Hunt, T.K., and Coughlin, S.R. 1997. Mice lacking the thrombin receptor, PAR1, have normal skin wound healing. Am. J. Pathol. 151:1199-1204.

50. Melis, E., et al. 2001. Targeted deletion of the cytosolic domain of tissue factor in mice does not affect development. Biochem. Biophys. Res. Commun. 286:580-586.

51. Ahamed, J., et al. 2006. Disulfide isomerization switches tissue factor from coagulation to cell signaling. Proc. Natl. Acad. Sci. U. S. A. 103:13932-13937.

52. Huang, M., et al. 1998. The mechanism of an inhibitory antibody on TF-initiated blood coagulation revealed by the crystal structures of human tissue factor, Fab 5G9 and TF.G9 complex. J. Mol. Biol. 275:873-894.

53. Mueller, B.M., Reisfeld, R.A., Edgington, T.S., and Ruf, W. 1992. Expression of tissue factor by melanoma cells promotes efficient hematogenous metastasis. Proc. Natl. Acad. Sci. U. S. A. 89:11832-11836.

54. Ruf, W., and Edgington, T.S. 1991. An anti-tissue factor monoclonal antibody which inhibits TF.VIIa complex is a potent anticoagulant in plasma. Thromb. Haemost. 66:529-533. 